\title{
WEAK LOG-MAJORIZATION OF UNITAL TRACE-PRESERVING COMPLETELY POSITIVE MAPS*
}

\author{
PAN-SHUN LAU ${ }^{\dagger}$ AND TIN-YAU TAM ${ }^{\dagger}$
}

\begin{abstract}
Let $\Phi: \mathbf{M}_{n} \rightarrow \mathbf{M}_{n}$ be a unital trace preserving completely positive map and $A \in \mathbf{M}_{n}$ be a positive definite matrix. Weak log-majorization and weak majorization between $\Phi(A)$ and $A$ are studied. Determinantal inequalities between $\Phi(A)$ and $A$ are obtained as a consequence. By considering special classes of unital trace preserving completely positive map, some known matrix inequalities such as Fischer's inequality are rediscovered. An affirmative answer to a question of Tam and Zhang in 2019 is given.
\end{abstract}

Key words. Completely positive maps, Weak log-majorization, Determinantal inequalities, Positive definite matrices.

AMS subject classifications. 15A42, $15 \mathrm{~A} 45$.

1. Introduction. Let $\mathbf{M}_{n}, \mathbb{P}_{n}$ and $\mathbb{P}_{n}^{+}$be the sets of all $n \times n$ complex matrices, positive semidefinite matrices and positive definite matrices, respectively. A linear map $\Phi: \mathbf{M}_{n} \rightarrow \mathbf{M}_{m}$ is positive if $\Phi\left(\mathbb{P}_{n}\right) \subseteq$ $\Phi\left(\mathbb{P}_{m}\right)$. Let $\mathbf{M}_{k}\left(\mathbf{M}_{n}\right)$ be the space of $k \times k$ block matrices $\left(A_{i j}\right)_{k \times k}$ where $A_{i j} \in \mathbf{M}_{n}$ for $1 \leq i, j \leq k$. For every linear map $\Phi: \mathbf{M}_{n} \rightarrow \mathbf{M}_{m}$, one can have an induced map $\Phi_{k}: \mathbf{M}_{k}\left(\mathbf{M}_{n}\right) \rightarrow \mathbf{M}_{k}\left(\mathbf{M}_{m}\right)$ defined as $\Phi_{k}\left(\left(A_{i j}\right)_{k \times k}\right)=\left(\Phi\left(A_{i j}\right)\right)_{k \times k}$. A linear map $\Phi: \mathbf{M}_{n} \rightarrow \mathbf{M}_{m}$ is completely positive if $\Phi_{k}$ is positive for all positive integers $k$. The concept of completely positive maps is introduced by Steinspring [23] in studying dilation problems of operators. In the landmark paper of Choi [6], he gave a complete characterization of completely positive maps, that is, $\Phi: \mathbf{M}_{n} \rightarrow \mathbf{M}_{m}$ is completely positive if and only if there are $n \times m$ complex matrices $F_{1}, \ldots, F_{r}$ such that

$$
\Phi(A)=\sum_{j=1}^{r} F_{j}^{*} A F_{j}
$$

Positive maps and completely positive maps are important concepts in operator algebra, quantum theory and matrix inequalities, see $[4,21]$ and their references.

A trace preserving completely positive map $\Phi$ is completely positive such that $\Phi(A)$ and $A$ have the same trace. Note the study of completely positive maps arises from the development of quantum information science. In the last few decades, fruitful results have been obtained by researchers, see [4, 10, 11, 16, 22]. Note that $\Phi$ is trace preserving if and only if the matrices $F_{1}, \ldots, F_{r}$ in (1.1) satisfy $\sum_{j=1}^{r} F_{j} F_{j}^{*}=I_{n}$.

A positive linear map $\Phi: \mathbf{M}_{n} \rightarrow \mathbf{M}_{m}$ is called unital if $\Phi\left(I_{n}\right)=I_{m}$. Unital positive linear maps are significant elements for enriching theories in the study of $C^{*}$-algebra. Note that a completely positive map $\Phi$ is unital if and only if the matrices $F_{1}, \ldots, F_{r}$ in (1.1) satisfy $\sum_{j=1}^{r} F_{j}^{*} F_{j}=I_{m}$. Moreover, $\Phi$ is unital if and only if its adjoint $\Phi^{*}$ is trace preserving. Some subclasses of unital trace preserving completely positive maps have been studied broadly by researchers such as mixed unitary maps and Schur product maps $[1,8,9,12,21,26]$.

\footnotetext{
*Received by the editors on August 16, 2019. Accepted for publication on September 13, 2019. Handling Editor: Michael Tsatsomeros. Corresponding Author: Pan-Shun Lau.

${ }^{\dagger}$ Department of Mathematics \& Statistics, University of Nevada, Reno, NV 89557-0084, USA (plau@unr.edu, ttam@unr.edu).
} 
For $x=\left(x_{1}, \ldots, x_{n}\right) \in \mathbb{R}^{n}$, we denote by $x^{\downarrow}=\left(x_{1}^{\downarrow}, \ldots, x_{n}^{\downarrow}\right)$ the vector obtained by rearranging the entries of $x$ in decreasing order, i.e., $x_{1}^{\downarrow} \geq \cdots \geq x_{n}^{\downarrow}$. For vectors $x, y \in \mathbb{R}^{n}, x$ is weakly majorized by $y$, denoted by $x \prec_{w} y$, if

$$
\sum_{j=1}^{k} x_{j}^{\downarrow} \leq \sum_{j=1}^{k} y_{j}^{\downarrow}, \quad 1 \leq k \leq n .
$$

In addition, $x$ is majorized by $y$, denoted by $x \prec y$, if (1.2) holds for $1 \leq k \leq n-1$ and equality holds when $k=n$. For positive vectors $x, y \in \mathbb{R}^{n}, x$ is weakly log-majorized by $y$, denoted by $x \prec_{w} \log y$, if

$$
\prod_{j=1}^{k} x_{j}^{\downarrow} \leq \prod_{j=1}^{k} y_{j}^{\downarrow}, \quad 1 \leq k \leq n .
$$

In other words, $x=\left(x_{1}, \ldots, x_{n}\right)$ is weakly $\log$-majorized by $y=\left(y_{1}, \ldots, y_{n}\right)$ if and only if the vector $\log (x)=$ $\left(\log x_{1}, \ldots, \log x_{n}\right)$ is weakly majorized by the vector $\log (y)=\left(\log y_{1}, \ldots, \log y_{n}\right)$. Weak majorization and majorization are powerful tools in studying matrix inequalities [3, 27]. Besides they have been widely applied in different areas such as number theory, statistics, numerical analysis, etc. see [13]. For any $n \times n$ Hermitian matrices $A$ and $B$, we say that $A \geq B$ if $A-B \in \mathbb{P}_{n}$. Moreover, we denote by $\lambda(A)=\left(\lambda_{1}(A), \ldots, \lambda_{n}(A)\right) \in \mathbb{R}^{n}$ where $\lambda_{1}(A), \ldots, \lambda_{n}(A)$ are eigenvalues of $A$ (including multiplicities). For $A, B \in \mathbb{P}_{n}$, we write $A \prec B$ if $\lambda(A) \prec \lambda(B)$. We define $A \prec_{w} B$ and $A \prec_{w \log } B$ accordingly.

The connection between majorization of Hermitian matrices and unital trace preserving completely positive maps is given by Uhlmann; see Proposition 2.2 below. Therefore, one may expect that techniques and tools in majorization can be applied to obtain or re-obtain insightful matrix inequalities for unital trace preserving completely positive maps. This is the main objective of this paper. In fact, by the works of Ando, Bhatia, Choi, and other researchers, one expects that the completely positive map is a powerful tool to study positive definite matrices and corresponding matrix inequalities; see [3] and its references.

In this paper, we focus on unital trace preserving completely positive maps $\Phi: \mathbf{M}_{n} \rightarrow \mathbf{M}_{n}$. In Section 2 , we study the weak log-majorization between $A \in \mathbb{P}_{n}^{+}$and $\Phi(A)$. We then strengthen the result to the sum of $A_{j}^{p_{j}}$ for positive definite matrices $A_{j}$ and real numbers $p_{j}, j=1, \ldots, m$. We will consider some special classes of unital trace preserving completely positive maps and answer in the affirmative an open problem in [24]. In Section 3, we apply the weak log-majorization obtained in Section 2 to study determinantal inequalities on positive semidefinite matrices. Similar inequalities have been studied by Nayak [18] under the context of von Neumann algebras and Fuglede-Kadison determinant. Some new inequalities are obtained as well as some known inequalities in [5, 20, 24]. In Section 4, we will make some remarks on the results obtained in Section 2.

2. Weak log-majorization. We start with the following lemma.

Lemma 2.1. Let $A, B \in \mathbb{P}_{n}^{+}$and let $B \prec A$, i.e., $\lambda(B) \prec \lambda(A)$. Then,

(a) $B^{-1} \prec w \log A^{-1}$;

(b) $I+B^{-1} \prec_{w \log } I+A^{-1}$.

Proof. Note that for $x=\left(x_{i}\right), y=\left(y_{i}\right) \in \mathbb{R}^{n}$, if $x \prec y$, then $\left(f\left(x_{i}\right)\right) \prec_{w}\left(f\left(y_{i}\right)\right)$ for any convex function $f: \mathbb{R} \rightarrow \mathbb{R}$, see [27, Theorem 10.12]. Hence, (a) and (b) follow from the convexity of the functions $f(x)=\log x^{-1}$ and $g(x)=\log \left(1+x^{-1}\right)$, respectively. 
The following result is one implication of a larger theorem known as Uhlmann's theorem [2], see also [11, Theorem 3.6].

Proposition 2.2. ([2]) Let $A \in \mathbb{P}_{n}$ and $\Phi: \mathbf{M}_{n} \rightarrow \mathbf{M}_{n}$ be a unital trace preserving completely positive map. Then $\Phi(A) \prec A$.

Combining Lemma 2.1 and Proposition 2.2, we have the following.

TheOREM 2.3. Let $A \in \mathbb{P}_{n}^{+}$and let $\Phi: \mathbf{M}_{n} \rightarrow \mathbf{M}_{n}$ be a unital trace preserving completely positive map. Then,

(a) $\Phi(A)^{-1} \prec_{w \log } A^{-1}$;

(b) $I+\Phi(A)^{-1} \prec_{w \log } I+A^{-1}$.

The following result is known and necessary for us to improve Theorem 2.3; see [4, Proposition 2.7.1 and Exercise 2.7.2].

Proposition 2.4. Let $A \in \mathbb{P}_{n}^{+}$and let $\Phi: \mathbf{M}_{n} \rightarrow \mathbf{M}_{n}$ be a unital positive map. Then,

$$
\Phi(A)^{p} \leq \Phi\left(A^{p}\right), \quad \text { where } p \in[-1,0] \cup[1,2],
$$

and

$$
\Phi(A)^{p} \geq \Phi\left(A^{p}\right), \quad \text { where } p \in[0,1] .
$$

We are ready to present our main result.

TheOREm 2.5. Let $A_{1}, \ldots, A_{m} \in \mathbb{P}_{n}^{+}$and let $\Phi: \mathbf{M}_{n} \rightarrow \mathbf{M}_{n}$ be a unital trace preserving completely positive map. Then, for $p_{1}, \ldots, p_{m} \in[-1,0]$,

$$
\sum_{j=1}^{m} \Phi\left(A_{j}\right)^{p_{j}} \prec_{w \log } \sum_{j=1}^{m} A^{p_{j}}
$$

and

$$
I+\sum_{j=1}^{m} \Phi\left(A_{j}\right)^{p_{j}} \prec w \log I+\sum_{j=1}^{m} A^{p_{j}} .
$$

Proof. Let $p_{1}, \ldots, p_{m} \in[-1,0]$. By Proposition 2.4, we have

$$
\Phi\left(A_{j}^{-p_{j}}\right) \leq \Phi\left(A_{j}\right)^{-p_{j}} \Rightarrow \Phi\left(A_{j}^{-p_{j}}\right)^{-1} \geq \Phi\left(A_{j}\right)^{p_{j}}
$$

where $j=1, \ldots, m$. Moreover, by the concavity of the map $A \mapsto \Phi\left(A^{-1}\right)^{-1}[4$, Exercise 4.5.3], we have

$$
\Phi\left(\left(\sum_{j=1}^{m} A_{j}^{p_{j}}\right)^{-1}\right)^{-1} \geq \sum_{j=1}^{m} \Phi\left(A_{j}^{-p_{j}}\right)^{-1} \geq \sum_{j=1}^{m} \Phi\left(A_{j}\right)^{p_{j}} .
$$

Replacing $A$ by $A^{-1}$ in Theorem 2.3 (a), we have $\Phi\left(A^{-1}\right)^{-1} \prec_{w} \log A$. Hence, by setting $A=\sum_{j=1}^{m} A^{p_{j}}$,

$$
\sum_{j=1}^{m} \Phi\left(A_{j}\right)^{p_{j}} \leq \Phi\left(\left(\sum_{j=1}^{m} A_{j}^{p_{j}}\right)^{-1}\right)^{-1} \prec_{w \log } \sum_{j=1}^{m} A^{p_{j}} .
$$

In addition, by similar argument and Theorem 2.3 (b), one can obtain the second weak log-majorization. 
One may ask if the assumptions on unital or trace-preserving in Theorem 2.5 can be dropped. However, for trace-preserving completely positive maps $\Phi: \mathbf{M}_{n} \rightarrow \mathbf{M}_{n}, \Phi(A)$ fails to be invertible in general for some positive definite $A$. On the other hand, the following example shows that Theorem 2.5 fails to hold if we remove the trace-preserving assumption.

Example 2.6. Let $A=\left[\begin{array}{ll}1 & 0 \\ 0 & 2\end{array}\right]$ and $\Phi: \mathbf{M}_{2} \rightarrow \mathbf{M}_{2}$ be defined by

$$
\Phi(X)=\left(\left[\begin{array}{ll}
1 & 0 \\
0 & 0
\end{array}\right] X\left[\begin{array}{ll}
1 & 0 \\
0 & 0
\end{array}\right]+\left[\begin{array}{ll}
0 & 0 \\
1 & 0
\end{array}\right] X\left[\begin{array}{ll}
0 & 1 \\
0 & 0
\end{array}\right]\right) .
$$

One can see from (1.1) that $\Phi$ is a completely positive map and $\Phi\left(I_{2}\right)=I_{2}$. However

$$
\Phi(A)^{-1}=I_{2} \nprec_{w \log }\left[\begin{array}{cc}
1 & 0 \\
0 & 1 / 2
\end{array}\right]=A^{-1} .
$$

Similarly, $I+\Phi(A)^{-1} \nprec_{w \log } I+A^{-1}$.

Moreover, if one considers unital trace-preserving positive maps, Theorem 2.3 fails to hold in general.

Example 2.7. Let $A=\left[\begin{array}{cc}1 & i \\ -i & 2\end{array}\right]$ and $\Phi: \mathbf{M}_{2} \rightarrow \mathbf{M}_{2}$ be defined by $\Phi(X)=\left(X+X^{t}\right) / 2$. Clearly, $\Phi$ is a unital trace-preserving positive map. Direct computation shows that $\Phi(A)^{-1}$ has eigenvalues $1 / 2,1$ and $A^{-1}$ has eigenvalues $0.3820,2.6180$. Hence, $\Phi(A)^{-1} \nprec_{w \log } A^{-1}$ and $I+\Phi(A)^{-1} \nprec_{w \log } I+A^{-1}$.

By combining Proposition 2.2 and Proposition 2.4, we have the following weak majorization for the case $p_{j} \in[-1,0] \cup[1,2], j=1, \ldots, m$.

Theorem 2.8. Let $A_{1}, \ldots, A_{m} \in \mathbb{P}_{n}^{+}$and let $\Phi: \mathbf{M}_{n} \rightarrow \mathbf{M}_{n}$ be a unital trace preserving completely positive map. Then, for $p_{j} \in[-1,0] \cup[1,2], j=1, \ldots, m$,

$$
\sum_{j=1}^{m} \Phi\left(A_{j}\right)^{p_{j}} \prec_{w} \sum_{j=1}^{m} A_{j}^{p_{j}} .
$$

Proof. By Proposition 2.2, we have

$$
\Phi\left(\sum_{j=1}^{m} A_{j}^{p_{j}}\right) \prec \sum_{j=1}^{m} A_{j}^{p_{j}} .
$$

Moreover, by Proposition 2.4, $\Phi\left(A_{j}\right)^{p_{j}} \leq \Phi\left(A_{j}^{p_{j}}\right), j=1, \ldots, m$. Hence,

$$
\sum_{j=1}^{n} \Phi\left(A_{j}\right)^{p_{j}} \leq \sum_{j=1}^{n} \Phi\left(A_{j}^{p_{j}}\right)=\Phi\left(\sum_{j=1}^{n} A_{j}^{p_{j}}\right) .
$$

Then the result follows.

Note that weak log-majorization implies weak majorization. Hence, if $p_{j} \in[-1,0]$ for all $j=1, \ldots, m$, then Theorem 2.8 follows from Theorem 2.5. However, the following example shows that Theorem 2.8 fails to hold if one replaces the weak majorization by weak log-majorization. It also shows that Theorem 2.5 fails to hold for $p_{j}>0, j=1, \ldots, m$. 
Example 2.9. Let $A=\left[\begin{array}{cc}3 / 2 & 0 \\ 0 & 1 / 2\end{array}\right]$ and $\Phi: \mathbf{M}_{2} \rightarrow \mathbf{M}_{2}$ be defined by

$$
\Phi(X)=\frac{1}{4}\left(\left[\begin{array}{cc}
1 & 1 \\
1 & -1
\end{array}\right] X\left[\begin{array}{cc}
1 & 1 \\
1 & -1
\end{array}\right]+\left[\begin{array}{cc}
1 & -1 \\
1 & 1
\end{array}\right] X\left[\begin{array}{cc}
1 & 1 \\
-1 & 1
\end{array}\right]\right) \text {. }
$$

One can see from (1.1) that $\Phi$ is a completely positive map. Moreover, it is clear that $\Phi$ is unital and trace preserving. Note that $\Phi(A)=I_{2}$. For every $p>0$, we have $1>\left(\frac{3}{2}\right)^{p} \cdot\left(\frac{1}{2}\right)^{p}$. Hence, $\Phi(A)^{p} \nprec_{w \log } A^{p}$.

A well-known subclass of unital trace preserving completely positive maps is called mixed unitary, i.e., $\Phi: \mathbf{M}_{n} \rightarrow \mathbf{M}_{n}$

$$
\Phi(X)=\sum_{j=1}^{r} t_{j} U_{j}^{*} X U_{j}
$$

where $U_{1}, \ldots, U_{r}$ are unitary matrices and $t_{1}, \ldots, t_{r}$ are nonnegative numbers summing up to 1 . They are also known as randomized unitary channels in quantum information.

For any $X \in \mathbf{M}_{n}$, write

$$
X=\left[\begin{array}{cccc}
X_{11} & X_{12} & \cdots & X_{1 r} \\
X_{21} & X_{22} & \cdots & X_{2 r} \\
\vdots & \vdots & \ddots & \vdots \\
X_{r 1} & X_{r 2} & \cdots & X_{r r}
\end{array}\right]
$$

where $X_{j j} \in \mathbf{M}_{n_{j}}, n_{1}+\cdots+n_{r}=n$. Let $w=e^{2 \pi i / r}$ and $V=I_{n_{1}} \oplus w I_{n_{2}} \oplus \cdots \oplus w^{r-1} I_{n_{r}}$. Then

$$
\Phi(X)=\frac{1}{r} \sum_{j=0}^{r-1} V^{* j} A V^{j}=X_{11} \oplus X_{22} \oplus \cdots \oplus X_{r r}
$$

is mixed unitary. Therefore, the following results are direct consequences of Theorem 2.5.

Corollary 2.10. Let $A_{j}=\left(A_{p q}^{(j)}\right)_{r \times r} \in \mathbb{P}_{n}^{+}$, where $A_{p p}^{(j)} \in \mathbf{M}_{n_{p}}, p_{j} \in[-1,0], j=1, \ldots, m$. Then

$$
\sum_{j=1}^{m}\left(A_{11}^{(j)}\right)^{p_{j}} \oplus \cdots \oplus \sum_{j=1}^{m}\left(A_{r r}^{(j)}\right)^{p_{j}} \prec_{w \log } \sum_{j=1}^{m} A_{j}^{p_{j}}
$$

and

$$
I+\sum_{j=1}^{m}\left(A_{11}^{(j)}\right)^{p_{j}} \oplus \cdots \oplus \sum_{j=1}^{m}\left(A_{r r}^{(j)}\right)^{p_{j}} \prec_{w \log } I+\sum_{j=1}^{m} A_{j}^{p_{j}} .
$$

Proof. Let $\Phi: \mathbf{M}_{n} \rightarrow \mathbf{M}_{n}$ be defined as (2.3). Then the result follows by direct application of Theorem 2.5 .

By setting $p_{j}=-1$ for $j=1, \ldots, m$ in Corollary 2.10, we have the following corollary which provides an affirmative answer to Question 3.8 in [24].

Corollary 2.11. Let $A_{j}=\left(A_{p q}^{(j)}\right)_{r \times r} \in \mathbb{P}_{n}^{+}$, where $A_{p p}^{(j)} \in \mathbf{M}_{n_{p}}, j=1, \ldots, m$. Then

$$
\sum_{j=1}^{m}\left(A_{11}^{(j)}\right)^{-1} \oplus \cdots \oplus \sum_{j=1}^{m}\left(A_{r r}^{(j)}\right)^{-1} \prec_{w \log } \sum_{j=1}^{m} A_{j}^{-1} .
$$


By setting $p_{j}=-1$ for $j=1, \ldots, m$ in Theorem 2.8, we have the following result which is first given in [24, Theorem 3.7].

Corollary 2.12. Let $A_{j}=\left(A_{p q}^{(j)}\right)_{r \times r} \in \mathbb{P}_{n}^{+}$, where $A_{p p}^{(j)} \in \mathbf{M}_{n_{p}}, p_{j} \in[-1,0] \cup[1,2], j=1, \ldots, m$. Then

$$
\sum_{j=1}^{m}\left(A_{11}^{(j)}\right)^{p_{j}} \oplus \cdots \oplus \sum_{j=1}^{m}\left(A_{r r}^{(j)}\right)^{p_{j}} \prec_{w} \sum_{j=1}^{m} A_{j}^{p_{j}}
$$

Proof. It follows from Theorem 2.8.

3. Determinantal inequalities. Weak log-majorization is a powerful tool to study determinantal inequalities of positive definite matrices. In this section, we will obtain some determinantal inequalities on unital trace-preserving completely positive maps. We start by a result of Olkin [20]. A correlation matrix is a positive semidefinite matrix and all its diagonal entries equal to one. It is known that if $X$ is a correlation matrix, then the map $\Phi_{X}: \mathbf{M}_{n} \rightarrow \mathbf{M}_{n}$ where $A \mapsto A \circ X$ is completely positive. Here, $A \circ X$ denotes the Schur product of $A$ and $X$. It is straightforward to see that $\Phi_{X}$ is unital and trace preserving. The following is a quick consequence of Theorem 2.3 on $\Phi_{X}$.

Proposition 3.1. Let $X$ be a correlation matrix and $A \in \mathbb{P}_{n}$. Then $\operatorname{det}(X \circ A) \geq \operatorname{det} A$.

Proof. By Theorem $2.3(\mathrm{a}),(X \circ A)^{-1} \prec w \log A^{-1}$. Hence, $\operatorname{det}(X \circ A)^{-1} \leq \operatorname{det} A^{-1}$. The result then follows.

Let $X=\left(x_{i j}\right)_{n \times n} \in \mathbf{M}_{n}$ be the correlation matrix, where $x_{i i}=1$ for all $i$, and $x_{i j}=\frac{1}{n(n-1)}$, for all $i \neq j$. For any correlation matrix $R \in \mathbf{M}_{n}$, Olkin [20] studied the average correlation matrix of $R$ defined as $\tilde{R}=X \circ R$. By Proposition 3.1, we rediscover the following result of Olkin. Note that the proof of Olkin makes use of permutation matrices and the fact that $\lambda(A+B) \prec \lambda(A)+\lambda(B)$ for Hermitian matrices $A, B$.

Corollary 3.2. Let $R \in \mathbb{P}_{n}$ be a correlation matrix and $\tilde{R}$ be the average correlation matrix of $R$. Then $\operatorname{det} \tilde{R} \geq \operatorname{det} R$.

The following result is a consequence from the weak log-majorization obtained in the previous section.

ThEOREM 3.3. Let $A_{1}, \ldots, A_{m} \in \mathbb{P}_{n}^{+}$and let $\Phi: \mathbf{M}_{n} \rightarrow \mathbf{M}_{n}$ be a unital trace preserving completely positive map. Then

$$
\operatorname{det}\left(\sum_{j=1}^{m} \Phi\left(A_{j}\right)^{p_{j}}\right) \geq \operatorname{det}\left(\sum_{j=1}^{m} A^{p_{j}}\right) \quad \text { for } p_{1}, \ldots, p_{m} \in[0,1]
$$

and

$$
\operatorname{det}\left(\sum_{j=1}^{m} \Phi\left(A_{j}\right)^{p_{j}}\right) \leq \operatorname{det}\left(\sum_{j=1}^{m} A^{p_{j}}\right) \quad \text { for } p_{1}, \ldots, p_{m} \in[-1,0] .
$$

Proof. Let $p_{j} \in[0,1], j=1, \ldots, m$. Theorem 2.3 (a) implies that $\operatorname{det} A \leq \operatorname{det} \Phi(A)$. By Proposition 2.4, we have

$$
\operatorname{det}\left(\sum_{j=1}^{m} A^{p_{j}}\right) \leq \operatorname{det}\left(\Phi\left(\sum_{j=1}^{m} A_{j}^{p_{j}}\right)\right)=\operatorname{det}\left(\sum_{j=1}^{m} \Phi\left(A_{j}^{p_{j}}\right)\right) \leq \operatorname{det}\left(\sum_{j=1}^{m} \Phi\left(A_{j}\right)^{p_{j}}\right)
$$

The second inequality follows from Theorem 2.5. 
By standard continuity argument, one sees that the first inequality of Theorem 3.3 holds for $A_{1}, \ldots, A_{m} \in$ $\mathbb{P}_{n}$.

In the following, we consider a class of completely positive maps called conditional expectation. It is introduced by Umegaki [25] in the context of von Neumann algebra. Here we focus on the algebra of $n \times n$ complex matrices $\mathbf{M}_{n}$ and a subalgebra $\mathbf{N}$ of $\mathbf{M}_{n}$.

Definition 3.4. A map $\Phi: \mathbf{M}_{n} \rightarrow \mathbf{N} \subseteq \mathbf{M}_{n}$ is called a conditional expectation if

(i) $\Phi$ is linear, positive, and unital, and

(ii) $\Phi(X Y Z)=X \Phi(Y) Z$ for any $X, Z \in \mathbf{N}$ and $Y \in \mathbf{M}_{n}$.

Note that every conditional expectation is a unital completely positive map; see [17]. Moreover, $\Phi(X)=$ $X$ for all $X \in \mathbf{N}$. We can improve the preceding results for trace preserving conditional expectation $\Phi: \mathbf{M}_{n} \rightarrow \mathbf{N}$ as follows.

TheOREm 3.5. Suppose $\Phi: \mathbf{M}_{n} \rightarrow \mathbf{N}\left(\subseteq \mathbf{M}_{n}\right)$ is a trace preserving conditional expectation. Let $A_{1}, \ldots, A_{m} \in \mathbb{P}_{n}^{+}$and $D_{1}, \ldots, D_{m} \in \mathbf{N}$. Then

$$
\operatorname{det}\left(\sum_{j=1}^{m} D_{i} \Phi\left(A_{j}\right)^{p_{j}} D_{i}^{*}\right) \geq \operatorname{det}\left(\sum_{j=1}^{m} D_{i} A^{p_{j}} D_{i}^{*}\right) \quad \text { for } p_{1}, \ldots, p_{m} \in[0,1]
$$

and

$$
\operatorname{det}\left(\sum_{j=1}^{m} D_{i} \Phi\left(A_{j}\right)^{p_{j}} D_{i}^{*}\right) \leq \operatorname{det}\left(\sum_{j=1}^{m} D_{i} A^{p_{j}} D_{i}^{*}\right) \quad \text { for } p_{1}, \ldots, p_{m} \in[-1,0] .
$$

Proof. Note that any positive map is $*$-preserving, i.e., $\Phi\left(T^{*}\right)=\Phi(T)^{*}$; see [4, Lemma 2.3.1]. Therefore, if $D \in \mathbf{N}$, then $\Phi\left(D^{*}\right)=\Phi(D)^{*}=D^{*}$ which implies that $D^{*} \in \mathbf{N}$.

For $p_{j} \in[0,1], j=1, \ldots, m$, by Theorem 2.3 (a) and Proposition 2.4, we have

$$
\operatorname{det}\left(\sum_{j=1}^{m} D_{i} A^{p_{j}} D_{i}^{*}\right) \leq \operatorname{det}\left(\Phi\left(\sum_{j=1}^{m} D_{i} A_{j}^{p_{j}} D_{i}^{*}\right)\right)=\operatorname{det}\left(\sum_{j=1}^{m} D_{i} \Phi\left(A_{j}^{p_{j}}\right) D_{i}^{*}\right) \leq \operatorname{det}\left(\sum_{j=1}^{m} D_{i} \Phi\left(A_{j}\right)^{p_{j}} D_{i}^{*}\right) .
$$

The second inequality can be shown similarly.

Note that the first inequality of Theorem 3.5 holds for $A_{1}, \ldots, A_{m} \in \mathbb{P}_{n}$.

Theorem 3.6. Suppose $\Phi: \mathbf{M}_{n} \rightarrow \mathbf{N}\left(\subseteq \mathbf{M}_{n}\right)$ is a trace preserving conditional expectation. Let $A \in \mathbb{P}_{n}^{+}$ and $D \in \mathbf{N}$. Then

$$
\frac{\operatorname{det}\left(\Phi(A)+D D^{*}\right)}{\operatorname{det}(\Phi(A))} \leq \frac{\operatorname{det}\left(A+D D^{*}\right)}{\operatorname{det} A} .
$$

Proof. We first assume that $D$ is invertible. As $D \in \mathbf{N}$,

$$
I=\Phi(I)=\Phi\left(D D^{-1}\right)=D \Phi\left(D^{-1}\right)
$$

Hence, $D^{-1}=\Phi\left(D^{-1}\right)$, that is, $D^{-1} \in \mathbf{N}$. Note that

$$
\frac{\operatorname{det}\left(\Phi(A)+D D^{*}\right)}{\operatorname{det}(\Phi(A))}=\operatorname{det}\left(I+D^{*} \Phi(A)^{-1} D\right)=\operatorname{det}\left(I+\Phi\left(D^{-1} A\left(D^{*}\right)^{-1}\right)^{-1}\right)
$$


and

$$
\frac{\operatorname{det}\left(A+D D^{*}\right)}{\operatorname{det} A}=\operatorname{det}\left(I+D^{*} A^{-1} D\right)=\operatorname{det}\left(I+\left(D^{-1} A\left(D^{*}\right)^{-1}\right)^{-1}\right) .
$$

The result follows by Theorem 2.3 (b). If $D$ is not invertible, then we may consider $D+\epsilon I$, where $\epsilon>0$. Clearly, we have $D+\epsilon I \in \mathbf{N}$ which is always invertible for small $\epsilon$. The result follows by a continuity argument.

Now consider the mixed unitary $\Phi$ defined in (2.3). Moreover, let $\mathbf{N}=\mathbf{M}_{n_{1}} \oplus \mathbf{M}_{n_{2}} \oplus \cdots \oplus \mathbf{M}_{n_{r}}$. Then $\Phi: \mathbf{M}_{n} \rightarrow \mathbf{N}$ is a trace preserving conditional expectation. We have the following.

Corollary 3.7. Let $A_{j}=\left(A_{p q}^{(j)}\right)_{r \times r} \in \mathbb{P}_{n}^{+}$, where $A_{p p}^{(j)} \in \mathbf{M}_{n_{p}}, j=1, \ldots, m$. Suppose $D_{j} \in \mathbf{M}_{n_{1}} \oplus$ $\mathbf{M}_{n_{2}} \oplus \cdots \oplus \mathbf{M}_{n_{r}}, j=1, \ldots, m$. Then

$$
\operatorname{det}\left(\sum_{j=1}^{m} D_{i}\left(A_{11}^{(j)} \oplus \cdots \oplus A_{r r}^{(j)}\right)^{p_{j}} D_{i}^{*}\right) \geq \operatorname{det}\left(\sum_{j=1}^{m} D_{i} A^{p_{j}} D_{i}^{*}\right) \quad \text { for } p_{1}, \ldots, p_{m} \in[0,1]
$$

and

$$
\operatorname{det}\left(\sum_{j=1}^{m} D_{i}\left(A_{11}^{(j)} \oplus \cdots \oplus A_{r r}^{(j)}\right)^{p_{j}} D_{i}^{*}\right) \leq \operatorname{det}\left(\sum_{j=1}^{m} D_{i} A^{p_{j}} D_{i}^{*}\right) \quad \text { for } p_{1}, \ldots, p_{m} \in[-1,0] .
$$

Proof. It follows from Theorem 3.5.

Note that the first inequality of Theorem 3.7 holds if $A_{1}, \ldots, A_{m} \in \mathbb{P}_{n}$. Corollary 3.7 is given in [5, Theorem 1.1 and Theorem 1.2] as a generalization of Fischer's inequality which is a particular case of Corollary 3.7 with $m=1, p_{1}=1$ and $D=I$.

Corollary 3.8. Let $A=\left(A_{p q}\right)_{r \times r}, C=\operatorname{diag}\left(C_{1}, \ldots, C_{r}\right) \in \mathbb{P}_{n}^{+}$, where $A_{p p}, C_{p} \in \mathbf{M}_{n_{p}}, p=1, \ldots, r$. Then

$$
\frac{\operatorname{det}(A+D)}{\operatorname{det} A} \leq \frac{\operatorname{det}\left(A_{11}+C_{1}\right)}{\operatorname{det} A_{11}} \ldots \frac{\operatorname{det}\left(A_{r r}+C_{r}\right)}{\operatorname{det} A_{r r}} .
$$

Proof. The result follows by Theorem 3.6 by setting $D=C^{1 / 2} \in \mathbf{N}$.

Corollary 3.8 is given in [14, Theorem 1.1]; see also [18].

4. Final remarks. Let $A, B \in \mathbf{M}_{n}$ and $\sigma(A), \sigma(B)$ be the set of singular values of $A$ and $B$, respectively. It is well-known that $\sigma(A) \prec_{w} \sigma(B)$ if and only if $\|A\| \leq\|B\|$ for all unitarily invariant matrix-vector norm $\|\cdot\|$ on $\mathbf{M}_{n}$, see [27, Theorem 10.38]. We end with the following result.

Theorem 4.1. Let $A_{1}, \ldots, A_{m} \in \mathbb{P}_{n}^{+}$and let $\Phi: \mathbf{M}_{n} \rightarrow \mathbf{M}_{n}$ be a unital trace preserving completely positive map. Then for all unitarily invariant matrix-vector norms $\|\cdot\|$ on $\mathbf{M}_{n}$ and $p_{1}, \ldots, p_{m} \in[-1,0]$,

$$
\left\|\sum_{j=1}^{m} \Phi\left(A_{j}\right)^{p_{j}}\right\| \leq\left\|\sum_{j=1}^{m} A^{p_{j}}\right\|
$$

and

$$
\left\|I+\sum_{j=1}^{m} \Phi\left(A_{j}\right)^{p_{j}}\right\| \leq\left\|I+\sum_{j=1}^{m} A^{p_{j}}\right\|
$$


Proof. The result follows by the fact that weak log-majorization implies weak majorization and Theorem 2.5.

Acknowledgment. We would like to thank the handling editor and an anonymous referee for comments and suggestions. We would also like to thank Prof. Chi-Kwong Li for some helpful discussion and comments.

\section{REFERENCES}

[1] K.M.R. Audenaert and S. Scheel. On random unitary channels, New J. Phys., 10:023011, 2008.

[2] P.M. Alberti and A. Uhlmann. Stochasticity and Partial Order: Doubly Stochastic Maps and Unitary Mixing. Springer, Dordrecht, 1982.

[3] R. Bhatia. Matrix Analysis. Springer-Verlag, New York, 1997.

[4] R. Bhatia. Positive Definite Matrices. Princeton University Press, Princeton, 2007.

[5] D. Choi, T.Y. Tam, and P. Zhang. Extension of Fischer's inequality. Linear Algebra Appl., 569:311-322, 2019.

[6] M.D. Choi. Completely positive linear maps on complex matrices. Linear Algebra Appl., 10:285-290, 1976.

[7] C. Davis. A Schwarz inequality for convex operator functions. Proc. Amer. Math. Soc., 8:42-44, 1957.

[8] S.J. Harris, R.H. Levene, V.I. Paulsen, S. Plosker, and M. Rahaman. Schur multipliers and mixed unitary maps. J. Math. Phys., 59:112201, 2018.

[9] J. Levick, D.W. Kribs, and R. Pereira. Quantum privacy and Schur product channels. Rep. Math. Phys., 80:333-347, 2017.

[10] C.K. Li and Y.T. Poon. Generalized numerical ranges and quantum error correction. J. Operator Theory, 66:335-351, 2011.

[11] C.K. Li and Y.T. Poon. Interpolation by completely positive maps. Linear Multilinear Algebra, 59:1159-1170, 2011.

[12] C.K. Li and H. Woerdeman. Special classes of positive and completely positive maps. Linear Algebra Appl., 255:247-258, 1997.

[13] A.W. Marshall, I. Olkin, and B.C. Arnold. Inequalities: Theory of Majorization and Its Applications, second edition. Springer, New York, 2011.

[14] I. Matic. Inequalities with determinants of perturbed positive matrices. Linear Algebra Appl., 449:166-174, 2014.

[15] C.B. Mendl and M.M. Wolf. Unital quantum channel's convex structure and revivals of Birkhoff's theorem. Comm. Math. Phys., 289:1057-1086, 2009.

[16] M. Nakahara and T. Ohmi. Quantum Computing: From Linear Algebra to Physical Realizations. CRC Press, Boca Raton, 2008.

[17] M. Nakamura, M. Takesaki, and H. Umegaki. A remark on the expectations of operator algebras. Kodai Math. Sem. Rep., 12:82-90, 1960.

[18] S. Nayak. The Hadamard determinant inequality - Extensions to operators on Hilbert space. J. Funct. Anal., 271:2978$3002,2018$.

[19] M.A. Nielsen and I.L. Chuang. Quantum Computation and Quantum Information. Cambridge University Press, Cambridge, 2000.

[20] I. Olkin. A determinantal inequality for correlation matrices. Statist. Probab. Lett., 88:88-90, 2014.

[21] V. Paulsen. Completely Bounded Maps and Operator Algebras. vCambridge University Press, Cambridge 2002.

[22] D. Petz. Quantum Information Theory and Quantum Statistics. Springer-Verlag, Berlin, 2008.

[23] W.F. Steinspring. Positive functions on $C^{*}$-algebras. Proc. Amer. Math. Soc., 6:211-216, 1955.

[24] T.Y. Tam and P. Zhang. Remarks on two determinantal inequalities. Math. Inequal. Appl., 22:815-823, 2019.

[25] H. Umegaki. Conditional expectation in an operator algebra. Tohoku Math. J., 6:177-181, 1954.

[26] J. Watrous. The Theory of Quantum Information. Cambridge University Press, Cambridge, 2018.

[27] F. Zhang. Matrix Theory: Basic Results and Techniques, second edition. Springer, New York, 2011. 